\title{
Bisphosphonate inhibits the expression of cyclin A2 at the transcriptional level in normal human oral keratinocytes
}

\author{
RACHEL S. LEE ${ }^{1}$, SUHJIN SOHN ${ }^{1}$, KI-HYUK SHIN ${ }^{1,2}$, MO K. KANG ${ }^{1,2}$, NO-HEE PARK ${ }^{1-3}$ and REUBEN H. KIM ${ }^{1,2}$ \\ ${ }^{1}$ The Shapiro Family Laboratory of Viral Oncology and Aging Research, UCLA School of Dentistry; \\ ${ }^{2}$ UCLA Jonsson Comprehensive Cancer Center; ${ }^{3}$ David Geffen School of Medicine at UCLA, Los Angeles, CA 90095, USA
}

Received April 23, 2016; Accepted May 29, 2017

DOI: $10.3892 /$ ijmm.2017.3066

\begin{abstract}
Nitrogen-containing bisphosphonates (N-BPs) are the most widely used anti-resorptive agents in the treatment of bonerelated diseases. N-BPs inhibit bone resorption by specifically targeting osteoclasts, bone-resorbing cells. However, soft tissue toxicity, such as oral or gastrointestinal (GI) ulcerations has frequently been reported in N-BP users, suggesting that N-BPs may also directly target cells other than osteoclasts. Previously, we reported that BPs inhibit proliferation without inducing the apoptosis of normal human oral keratinocytes (NHOKs). However, the molecular mechanisms through which N-BPs inhibit the proliferation of NHOKs are not yet fully understood. In this study, we performed gene expression profiling in N-BP-treated NHOKs and identified cyclin A2 as one of the most commonly downregulated genes. When the NHOKs were treated with N-BPs, we found that the level of cyclin A2 was suppressed in a dose- and time-dependent manner. In addition, the protein level of cyclin A2 was also significantly lower in oral epithelial cells in N-BP-treated oral mucosal tissue constructs. Cyclin A2 promoter reporter assay revealed that N-BPs inhibited the luciferase activity, indicating that the inhibition of cyclin A2 expression occurs at the transcriptional level. Furthermore, N-BPs did not alter the expression of cyclin A2 in normal human oral fibroblasts (NHOFs), suggesting that the effect of N-BPs on cyclin A2 expression may be cell-type specific. Thus, the findings of our study demonstrate that the inhibition of NHOK proliferation by N-BPs is mediated, at least in part, by the suppression of cyclin A2 expression at the transcriptional level, which may explain the underlying mechanisms of soft tissue toxicity by N-BPs.
\end{abstract}

\section{Introduction}

Nitrogen-containing bisphosphonates (N-BPs) are the most commonly used anti-resorptive agents in the treatment of

Correspondence to: Professor Reuben H. Kim, The Shapiro Family Laboratory of Viral Oncology and Aging Research, UCLA School of Dentistry, Room 43-091, 10833 Le Conte Ave, Los Angeles, CA 90095, USA

E-mail: rkim@dentistry.ucla.edu

Key words: bisphosphonate, cyclin A2, oral keratinocytes bone-related diseases, such as osteoporosis and metastatic bone diseases (1). Due to their ability to bind and chelate calcium, $\mathrm{N}$-BPs are preferentially localized to the bone matrix comprised of calcium-enriched inorganic hydroxyapatite (2). N-BPs exert their anti-resorptive effects by nullifying the functions of osteoclasts, the only bone-resorbing cells in the body; N-BPs are taken up by osteoclasts, as these cells resorb bone. Clinical studies suggest that N-BPs have side-effects on tissues other than bone, such as epithelial tissues (3). Indeed, gastrointestinal (GI) toxicity is one of the major side-effects of BP and a leading cause of therapy termination during N-BP clinical trials (4). Esophageal/oral inflammation and ulceration are frequently noted in N-BP-users $(5,6)$. These studies suggest that N-BP may have direct effects on epithelial cells.

The epithelium is also known to be highly enriched in calcium contents, such that the concentration of calcium gradually increases from the basal to the outer layers (7). We previously found that N-BPs localize not only to bone, but also to the epithelium, as demonstrated by fluorescently-labeled N-BPs in osteomucosal tissue constructs (8). We also reported that N-BPs inhibit proliferation by inducing cell cycle arrest at the $\mathrm{S}$ phase without inducing the apoptosis of normal human oral keratinocytes (NHOKs) (9). Nonetheless, the molecular mechanisms underlying the suppressive effects of N-BPs on the proliferation of keratinocytes remain unclear.

In our continual efforts to elucidate the molecular mechanisms of N-BP on NHOKs, in this study, we performed gene expression profiling with DNA microarray on NHOKs treated with either pamidronate (PAM) or zoledronate (ZOL), two of which are the most frequently used N-BPs in clinical practice. Using this high-throughput approach, we identified and validated cyclin A2 as one of the bisphosphonate target genes associated with $\mathrm{S}$ phase cell cycle arrest and the inhibition of cell proliferation.

\section{Materials and methods}

Cells and cell culture. Primary NHOKs and normal human oral fibroblasts (NHOFs) were obtained according to previously described methods (9) following the approval of the Institutional Review Board (IRB\# 04-060-04). Briefly, discarded oral mucosal tissues were collected without identifiers from the normal patients who are undergoing routine dental procedures (e.g., gingivectomy for the crown-lengthening 
Table I. Primer sequences used for PCR.

\begin{tabular}{|c|c|c|}
\hline Gene & Forward primer & Reverse primer \\
\hline $\mathrm{CDC} 2$ & 5 '-tggatctgaagaaatacttggattcta-3' & 5'-caatcccctgtaggatttgg-3' \\
\hline CDC6 & 5'-cctgttctcctcgtgtaaaagc-3' & 5'-gtgttgcataggttgtcatcg-3' \\
\hline $\mathrm{CDC} 20$ & 5'-cattcgcatctggaatgtgt-3' & 5'-gagaccagaggatggagcac-3' \\
\hline $\mathrm{E} 2 \mathrm{~F} 8$ & 5 '-aatgacatctgccttgacga-3' & $5^{\prime}$-gtaaatgcgtcgacgttcaa-3' \\
\hline CCNA2 & $5^{\prime}$-ggtactgaagtccgggaacc-3' & 5 '-gaagatccttaaggggtgcaa-3' \\
\hline CCNB1 & $5^{\prime}$-catggtgcactttcctcctt-3' & 5'-aggtaatgttgtagagttggtgtcc-3 \\
\hline MMP10 & 5'-caaaagaggaggactccaaca-3' & 5'-ttcacatccttttcgaggttg-3' \\
\hline LCE3D & 5'-ctcctctgcacctggacaa-3' & 5'-cacttgggtgagggacactt-3' \\
\hline LCE3E & 5'-acgcatgccttcccatatac-3' & 5'-gagctcagatcccccacag-3' \\
\hline MAFB & 5'-gcaggtataaacgcgtccag-3' & 5'-tgaatgagctgcgtcttctc-3' \\
\hline IFIT1 & 5'-tggcagaagcccagacttac-3' & $5^{\prime}$-agggatttgaaagcttcttgc-3' \\
\hline SLITRK6 & 5'-agctccagcetgatatggag-3' & $5^{\prime}$-ttccattaacttcagctcttcgt-3' \\
\hline
\end{tabular}

procedures). The obtained oral mucosal tissues were cut into $25 \mathrm{~mm}^{2} \times 0.5 \mathrm{~mm}$ sections and incubated in $2.5 \mathrm{mg} / \mathrm{ml}$ dispase solution (Cat. no. 17105; Gibco, Grand Island, NY, USA) in $37^{\circ} \mathrm{C}$ for $1 \mathrm{~h}$, after which the epithelial layers and connective layers were separated. The separated epithelial layers were minced and subjected to trypsin digestion (Cat. no. 25200056; Gibco) in $37^{\circ} \mathrm{C}$ for 5 min to harvest the NHOKs. The separated connective tissue layers were minced and subjected to collagenase digestion (Cat. no. 17100017; Gibco) in $37^{\circ} \mathrm{C}$ forl h to harvest the NHOFs. The NHOKs were cultured in EpiLife supplemented with human keratinocyte growth supplement (HKGS) (Cascade Biologics, Portland, OR, USA), and the NHOFs were cultured in DMEM supplemented with 10\% FBS (Invitrogen, Carlsbad, CA, USA). ZOL and PAM were purchased from LKT Laboratories, Inc. (St. Paul, MN, USA). Throughout the study, 1,2 , and $4 \mu \mathrm{M}$ of ZOL and 10, 20 and $50 \mu \mathrm{M}$ of PAM were used to treat the cells for the indicated periods of time.

DNA microarray. Gene expression profiling was performed using the Affymetrix GeneChip Human Genome U133 Plus 2.0 Array (Affymetrix, Santa Clara, CA, USA). Total RNA was extracted from the cells using the RNeasy mini kit (Qiagen, Hilden, Germany) according to the manufacture's instructions. RNA integrity was evaluated using an Agilent 2100 Bioanalyzer (Agilent Technologies, Palo Alto, CA, USA) and the purity/ concentration was determined using a NanoDrop 8000 spectrophotometer (NanoDrop Products, Wilmington, DE, USA). Microarray experiments were performed at UCLA Clinical MIcroarray Core (CMC). Microarray targets were prepared using NuGEN WT-Ovation Formalin-Fixed Paraffin-Embedded RNA Amplification System and FL-Ovation cDNA Biotin Module V2 (NuGEN Technologies, San Carlos, CA, USA) and then hybridized to the array, all according to the manufacturers' instructions. The arrays were washed and stained with streptavidin phycoerythrin in Affymetrix Fluidics Station 450 using the Affymetrix GeneChip protocol, and then scanned using an Affymetrix GeneChip Scanner 3000. The acquisition and initial quantification of array images were conducted using the AGCC software (Affymetrix). The subsequent data analyses were performed using Partek Genomics Suite Version 6.4 (Partek, St. Louis, MO, USA). Differentially expressed genes were selected at $\geq 2$-fold and $P<0.005$. Ingenuity Pathway Analysis was also performed by the UCLA CMC laboratory.

Conventional and reverse transcription-quantitative $P C R(R T-q P C R)$. Total RNA was isolated from the cultured cells using TRIzol ${ }^{\mathrm{TM}}$ reagent (Invitrogen) and was then subjected to RNases-free DNase I digestion at $37^{\circ} \mathrm{C}$ for $2 \mathrm{~h}$ to eliminate any genomic DNA contamination. DNA-free total RNA $(5 \mu \mathrm{g})$ was dissolved in $15 \mu \mathrm{l}$ diethylpyrocarbonate-treated water, and the reverse transcription reaction was performed in First-Strand buffer containing 300 U SuperScript II (both from Invitrogen) with $10 \mathrm{mM}$ DTT and $0.5 \mu \mathrm{g}$ random hexamer (Promega, Madison, WI, USA) and $125 \mu \mathrm{M}$ deoxynucleoside triphosphates. The annealing reaction was carried out for $5 \mathrm{~min}$ at $65^{\circ} \mathrm{C}$, and complementary DNA synthesis was performed for $2 \mathrm{~h}$ at $37^{\circ} \mathrm{C}$, followed by incubation for $15 \mathrm{~min}$ at $70^{\circ} \mathrm{C}$ to terminate the enzyme reaction. The reverse transcription product was diluted with $70 \mu \mathrm{l}$ $\mathrm{H}_{2} \mathrm{O}$. Conventional PCR was performed in triplicate for each $2 \mu \mathrm{l}$ cDNA and the resultant PCR amplicants were visualized by running the agarose gel. Quantitative (real-time) (qPCR) was performed in triplicate for each $1 \mu 1 \mathrm{cDNA}$ sample with LC480 SYBR-Green I master using universal cycling conditions on LightCycler 480 from Roche (Indianapolis, IN, USA). A total of 45 cycles were executed, and the second derivative $\mathrm{Cq}$ value determination method was used to compare fold differences. The primer sequences were obtained from the Universal Probe Library (Roche). The following primers were used for PCR amplification: glyceraldehyde 3-phosphate dehydrogenase (GAPDH) primers, 5'-GAC CCC ATT GAC CTC AAC-3' (forward) and 5'-CTT CTC CAT GGT GGT GAA GA-3' (reverse); and cyclin A2 primers, 5'-GAG GAC CAG GAG AAT ATC AAC CCG G-3' (forward) and 5'-AGC CAG GGC ATC TTC ACG CTC TAT T-3' (reverse). RT-qPCR was also performed to confirm the data obtained from microarray analyses as described above and using the primer sequences listed in Table I. 
Western blot analysis. The cells were lysed and subjected to western blot analysis as previously described (9). Briefly, the cells were washed twice with PBS prior to treatment with ice-cold lysis buffer (20 mM Tris- $\mathrm{HCl}, \mathrm{pH} 7.4,150 \mathrm{mM}$ $\mathrm{NaCl}, 5 \mathrm{mM}$ EDTA and 1\% Triton X-100). The cells were then scraped and incubated on ice for $10 \mathrm{~min}$. Cell debris was separated by centrifugation at $20,000 \mathrm{x} \mathrm{g}$ at $4^{\circ} \mathrm{C}$ for $20 \mathrm{~min}$ and the supernatant was collected for western blot analysis after being subjected to 8 or $10 \%$ SDS-PAGE. Following electrophoresis, the proteins were transferred onto immobilized membranes (Millipore, Billerica, MA, USA), which were subsequently blocked with $5 \%$ non-fat milk for $1 \mathrm{~h}$ at room temperature. The membranes were then incubated with primary antibodies, and probed with the respective secondary antibodies conjugated with HRP. The signals were obtained using ChemiDoc XRS system (Bio-Rad, Hercules, CA, USA). The following primary antibodies were used: cyclin A2 (C-19), cyclin D1 (C-20; sc-717), Cdk6 (C-21; sc-117), Cdk2 (M2; sc-163) and $\beta$-actin (I-19; sc-1616) (all from Santa Cruz Biotechnology, Inc., Santa Cruz, CA, USA); and proliferating cell nuclear antigen (PCNA) (Ab-1; NA03-200UG) from Oncogene Research Product (Boston, MA, USA).

Analysis of BP-responsive reporter activity. The cyclin A2promoter luciferase reporter vector (pGL3-cyclinA2p) was constructed by inserting a region of the cyclin A2 promoter $(-516$ to +92$)$ into the pGL3-basic plasmid (Promega). Briefly, the cyclin A2 promoter was PCR-amplified from the pALUCcyclin A2 reporter plasmid, kindly provided by Dr Berthold Henglein (Institut National de la Santé et de la Recherche Médicale, Paris, France). The 608 bp cyclin A 2 promoter region was cloned into the cloning vector, cut out by double-digesting with SacI (New England Biolabs, Beverly, MA, USA) and XhoI (Invitrogen), and ligated into the pGL3-basic plasmid. The correct sequence was confirmed using sequencing analysis. The cells were transfected with $1 \mu \mathrm{g}$ of either pGL3-basic or pGL3-cyclinA2p using Lipofectamine 2000 (Invitrogen). The pRL-SV40 plasmid (0.005 $\mu \mathrm{g} /$ well) containing the Renilla luciferase gene driven by the SV40 promoter was co-transfected as a control. After $48 \mathrm{~h}$, the cells were harvested, and luciferase activity was measured using the Dual Luciferase Reporter assay system using a luminometer (both from Promega). The experiment was performed in triplicate.

Organotypic 3-dimensional (3D) raft cultures. The cells were grown as organotypic raft cultures using previously established techniques (9). Briefly, $1 \times 10^{6}$ cells were seeded on the submucosal equivalents consisting of type I collagen and NHOFs. The cells were grown to confluence, submerged in culture medium, and then exposed to the liquid-air interface by lowering the medium level. The cultures were maintained in this 'rafting' fashion for 14 days and were harvested by fixing in $10 \%$ buffered formalin. Subsequently, hematoxylin and eosin (H\&E) staining was performed on 6- $\mu$ m-thick sagittal sections of each reconstructs to reveal the histological features. Sample processing, paraffin embedding, sectioning and H\&E staining were performed at the Translational Pathology Core Laboratory at UCLA.

Immunohistochemical (IHC) staining. IHC staining was performed on the organotypic raft culture samples as previ- ously described (9). Cyclin A2 expression was determined in bisphosphonate-treated or -untreated oral mucosal tissue samples from raft cultures, which were formalin-fixed, paraffinembedded and sectioned. The specimens were subjected to IHC analysis for the expression of cyclin A2 (C-19; Santa Cruz Biotechnology, Inc.) at 1:200 concentrations. Stained tissues were developed using the 3,3'-diaminobenzidine (DAB) chromogen substrate (Vector Laboratories, Inc., Burlingame, CA, USA). The samples were counterstained with hematoxylin.

Statistical analysis. The results are expressed as the means \pm standard deviation. For the comparison, the outcome measurements were compared to the control group using the Student's t-test. Values of $\mathrm{p}<0.05$ are considered significant.

\section{Results}

Gene expression profiling in ZOL or PAM-treated NHOKs. Previously, we demonstrated that N-BPs inhibit proliferation without causing the apoptosis of NHOKs (9). In this study, to elucidate the mechanisms through which N-BPs inhibit cell proliferation, we performed gene expression profiling using NHOKs treated with PAM or ZOL at concentrations that cause a significant loss of proliferation (data not shown). We used various concentrations of PAM and ZOL as ZOL is 2-4-fold more potent than PAM (10). In the ZOL-treated NHOKs, 480 genes were differentially expressed, whereas 383 genes were differentially expressed in the PAM-treated NHOKs when compared to the untreated NHOKs (Fig. 1A). From these data, we identified 280 genes that were commonly present in both the ZOL- and PAM-treated NHOKs (Fig. 1A). Ingenuity Pathway Analysis (IPA) revealed that, of these 280 genes, many of them were associated with cell cycle control of chromosomal replication (Fig. 1B). Indeed, genes related to cell cycle regulation, such as cell division cycle(CDC)20, CDC6, cyclin (CCN) A2, CCNB1, E2F transcription factor 8 (E2F8) and CDC2, were downregulated by the bisphosphonates, whereas genes that are more involved in structural formation, such as matrix metallopeptidase 10 (MMP10), late cornified envelope 3E (LCE3E), MAF BZIP transcription factor B (MAFB), SLIT and NTRK like family member 6 (SLITRK6), late cornifiedenvelope 3D (LCE3D) and interferon induced protein with tetratricopeptide repeats 1 (IFIT1), exhibited an increased expression (Table II and Fig. 1C). To confirm the data from the microarray analysis, we performed RT-qPCR and found consistent results (Fig. 1D and E), supporting the validity of the microarray data.

$N$-BPs inhibit the expression of cyclin A2. As previously demonstrated, when NHOKs were treated with the BPs, they underwent cell cycle arrest at the $S$ phase $(9,11)$. As our microarray data revealed the effects of N-BPs on genes associated with the cell cycle, we screened for cell cyclerelated proteins. When the NHOKs were treated with PAM, the expression of PCNA, a proliferation marker, decreased in time- and dose-dependent manner (Fig. 2A). Consistent with the microarray data, the expression of cyclin A2, a protein that is associated with the $\mathrm{S}$ phase in the cell cycle, was markedly diminished to an undetectable level by PAM. The dose-dependent decrease in cyclin A2 expression was also 
Table II. Differentially expressed genes by N-BPs (fold induction).

\begin{tabular}{|c|c|c|c|c|}
\hline Gene symbol & Gene title & CTL vs PAM/ZOL & CTL vs PAM & CTL vs ZOL \\
\hline CLDN11 & Claudin 11 & -7.48 & -7.34 & -7.62 \\
\hline $\mathrm{CDC} 20$ & Cell division cycle 20 homolog & -4.59 & -3.77 & -5.58 \\
\hline CDC6 & Cell division cycle 6 homolog & -4.08 & -3.83 & -4.36 \\
\hline CDKN3 & Cyclin-dep kinase inhibitor 3 & -3.89 & -3.37 & -4.49 \\
\hline LMNB1 & Lamin B1 & -3.82 & -3.41 & -4.27 \\
\hline $\mathrm{CCNA} 2$ & Cyclin A2 & -3.78 & -3.53 & -4.05 \\
\hline CCNB1 & Cyclin B1 & -3.44 & -3.14 & -3.77 \\
\hline EPCAM & Epithelial cell adhesion molecule & -3.44 & -3.64 & -3.24 \\
\hline E2F8 & E2F transcription factor 8 & -3.37 & -3.24 & -3.51 \\
\hline HMGB2 & High-mobility group box 2 & -3.1 & -2.95 & -3.26 \\
\hline $\mathrm{CDC} 2$ & Cell division cycle 2 & -2.87 & -3.03 & -2.71 \\
\hline FOXM1 & Forkhead box M1 & -2.72 & -2.6 & -2.85 \\
\hline HNRNPD & Hn ribonuclearprotein $\mathrm{D}$ & -2.57 & -2.3 & -2.88 \\
\hline CDKN2C & Cyclin-dep kinase inhibitor $2 \mathrm{C}$ & -2.5 & -2.32 & -2.68 \\
\hline RBL1 & Retinoblastoma-like 1 (p107) & -2.28 & -2.31 & -2.25 \\
\hline FOXA2 & Forkhead box A2 & -2.26 & -2.37 & -2.15 \\
\hline BRCA2 & Breast cancer 2, early onset & -2.25 & -2.31 & -2.2 \\
\hline RAD51 & RAD51 homolog & -2.21 & -2.12 & -2.3 \\
\hline MSH2 & musS homolog 2 & -2.18 & -2.17 & -2.18 \\
\hline $\mathrm{EZH} 2$ & Enhancer of zeste homolog 2 & -2.18 & -2.17 & -2.18 \\
\hline RAD54B & RAD54 homolog B & -2.16 & -2.15 & -2.17 \\
\hline LCE3E & Late cornified envelope $3 \mathrm{E}$ & 1.79 & 1.57 & 2.05 \\
\hline SLITRK6 & $\begin{array}{l}\text { SLIT and NTRK-like family, } \\
\text { member } 6\end{array}$ & 2.08 & 1.88 & 2.29 \\
\hline TP53INP2 & p53 inducible nuclear protein 2 & 2.13 & 2.01 & 2.24 \\
\hline $\mathrm{CD} 24$ & CD24 molecule & 2.19 & 2.22 & 2.16 \\
\hline TNFSF10 & TNF superfamily member 10 & 2.43 & 2.45 & 2.4 \\
\hline MMP10 & Matrix metallopeptidase 10 & 2.24 & 2.25 & 2.22 \\
\hline MAFB & $\begin{array}{l}\text { V-maf musculoaponeurotic } \\
\text { fibrosarcoma oncogene homolog B }\end{array}$ & 2.31 & 2.38 & 2.24 \\
\hline TP53INP1 & p53 inducible nuclear protein 1 & 2.37 & 2.35 & 2.39 \\
\hline SOX4 & SRY-box 4 & 2.59 & 2.63 & 2.54 \\
\hline LCE3D & Late cornified envelope 3D & 2.65 & 2.68 & 2.63 \\
\hline FN1 & Fibronectin 1 & 2.9 & 2.7 & 3.11 \\
\hline IFIT1 & $\begin{array}{l}\text { Interferon-induced protein } \\
\text { with tetratricopeptide repeats } 1\end{array}$ & 2.93 & 3.53 & 2.43 \\
\hline IL6 & Interleukin 6 & 3.34 & 2.95 & 3.79 \\
\hline
\end{tabular}

N-BPs, nitrogen-containing bisphosphonates; PAM, pamidronate; ZOL, zoledronate.

observed in the ZOL-treated NHOKs (Fig. 2B). Previously, we also demonstrated that BPs induce inhibitory effects on epithelial tissues using oral mucosal tissue constructs (9). To examine whether the anti-proliferative effects of BPs on epithelial tissues are associated with cyclin A2 expression, we treated the oral mucosal tissue constructs with ZOL. As expected, tissue atrophy with thinning of the epithelial layers was observed (Fig. 2C). We then performed IHC staining for cyclin A2 in the ZOL-treated oral mucosal tissue constructs. The results revealed a marked reduction in cyclin A2 staining patterns in the epithelial layer of the ZOL-treated tissues (Fig. 2D). Collectively, these data indicate that the suppression of cell proliferation at the $\mathrm{S}$ phase is associated with the suppression of cyclin A2 expression.

$N$-BPs inhibit cyclin A2 expression at the transcriptional level. To further examine the molecular mechanisms through which bisphosphonates regulate the expression of cyclin A2, we examined expression of cyclin A2 at the transcriptional level. Similar to the results of western blot analysis, the mRNA level of cyclin A2 decreased in a time- and dose-dependent manner (Fig. 3A). To further confirm this finding, we constructed 

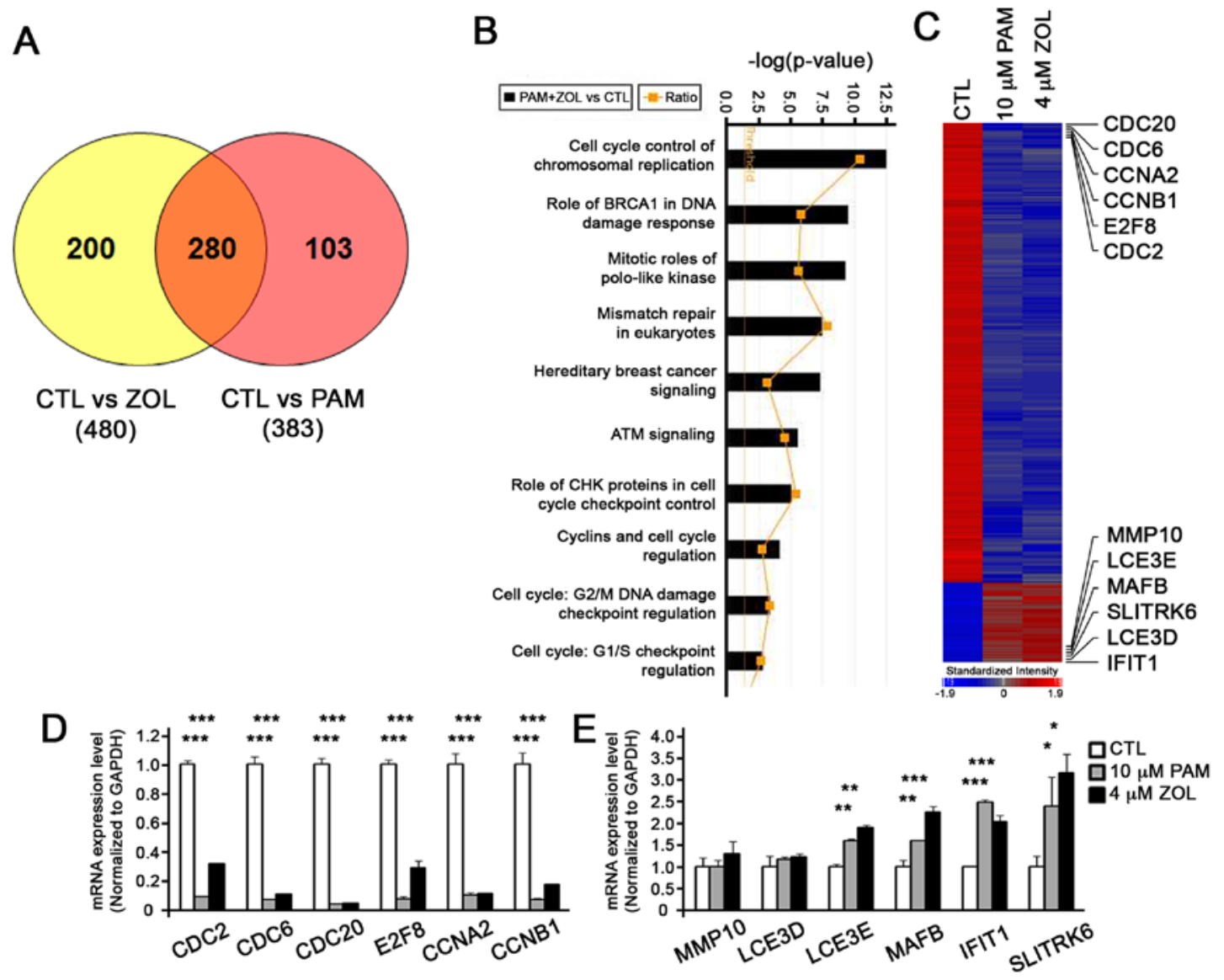

Figure 1. Gene expression profiling in zoledronate (ZOL)- or pamidronate (PAM)-treated normal human oral keratinocytes (NHOKs). (A) NHOKs were treated without or with $10 \mu \mathrm{M}$ PAM or $4 \mu \mathrm{M} Z$ ZOL for 4 days $(\mathrm{n}=3)$, and subjected to gene expression profiling using DNA microarray. Venn diagram of the differentially regulated genes by ZOL or PAM. (B) Ingenuity Pathway Analysis (IPA) of genes commonly regulated by both ZOL and PAM. (C) Heatmap representing the decreased gene expression (top of heatmap) and increased gene expression (bottom of heatmap) in N-BP-treated NHOKs compared to the controls. NHOKs were treated with $4 \mu \mathrm{M} \mathrm{ZOL}$ or $10 \mu \mathrm{M}$ PAM for 4 days and RT-qPCR was performed to validate genes that are (D) downregulated or (E) upregulated. Gene expression was normalized to GAPDH. ${ }^{*} \mathrm{p}<0.05 ;{ }^{* *} \mathrm{p}<0.01 ;{ }^{* * *} \mathrm{p}<0.001$.

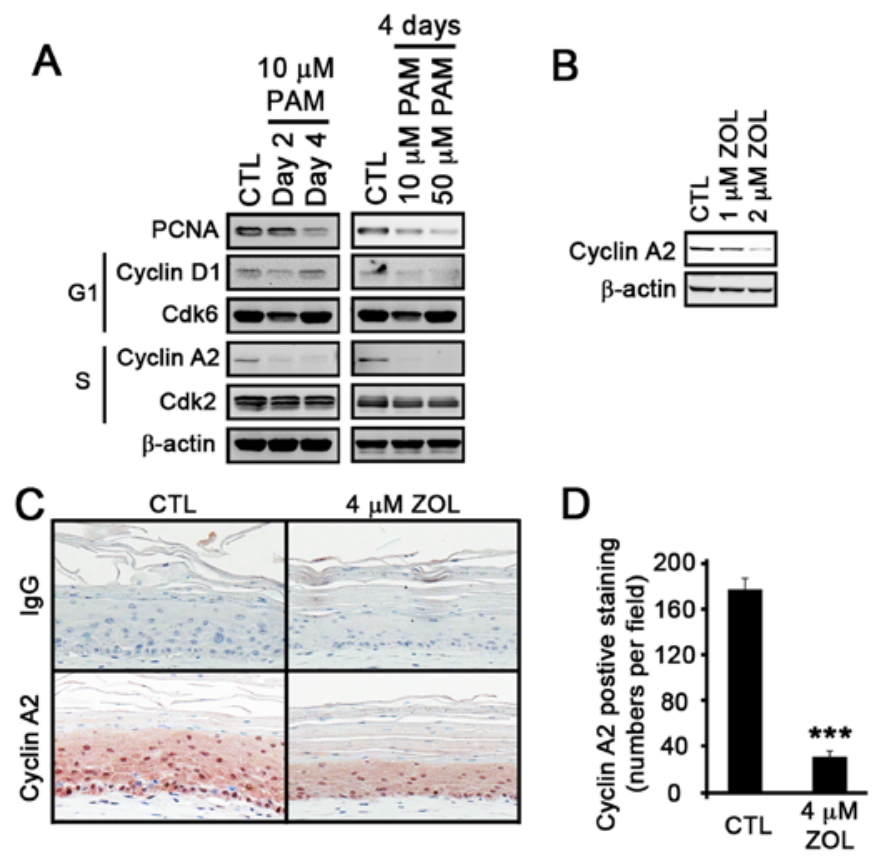

Figure 2. Nitrogen-containing bisphosphonates (N-BPs) inhibit the expression of cyclin A2. (A) Normal human oral keratinocytes (NHOKs) were treated with $10 \mu \mathrm{M}$ pamidronate (PAM) for 2 or 4 days. NHOKs were also treated with 10 or $50 \mu \mathrm{M}$ PAM for 4 days. Cell lysates were subjected to western blot analysis for PCNA, cyclin D1, Cdk6, cyclin A2, Cdk2 and $\beta$-actin. (B) NHOKs were treated with 1 or $2 \mu \mathrm{M}$ zoledronate (ZOL) and cyclin A2 expression was examined by western blot analysis. (C) Oral mucosal tissues were constructed, and $4 \mu \mathrm{M}$ ZOL was treated for 7 days. Immunohistochemical staining was performed using cyclin A2 antibody (1:200). IgG was used as a negative control. (D) The cyclin A2 staining pattern in the epithelial tissue layers were quantified by counting DAB-positive cells in 3 independent fields of oral mucosal tissue constructs. ${ }^{* * *} \mathrm{p}<0.001$. 

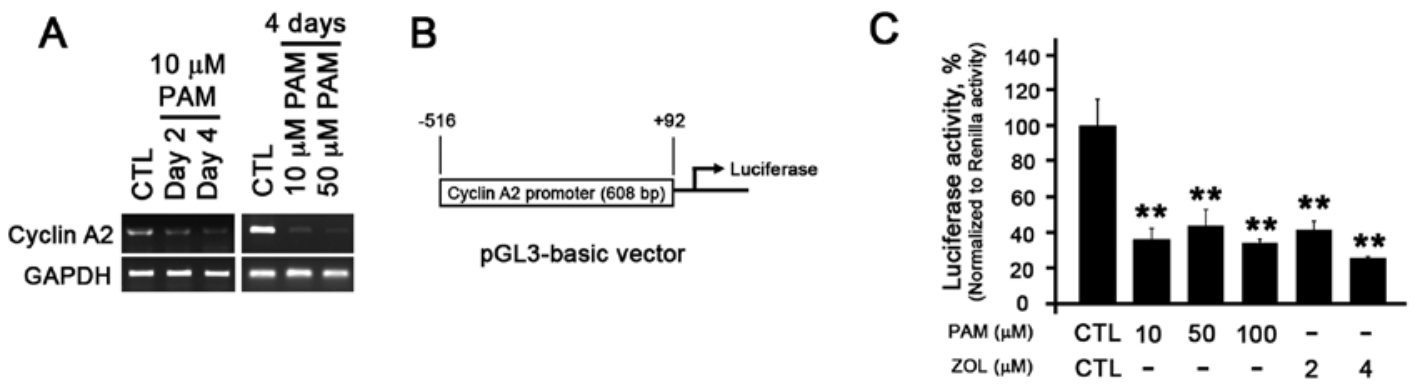

Figure 3. Nitrogen-containing bisphosphonates (N-BPs) inhibit cyclin A2 expression at the transcriptional level. (A) Normal human oral keratinocytes (NHOKs) were treated with $10 \mu \mathrm{M}$ pamidronate (PAM) for 2 or 4 days. NHOKs were also treated with 10 or $50 \mu \mathrm{M}$ PAM for 4 days. Cyclin A2 expression was examined by RT-qPCR. (B) Cyclin A2 luciferase reporter vector was constructed by cloning the cyclin A2 promoter region (-516 to +92) into the pGL3-basic vector. (C) Luciferase reporter assay was conducted by transfecting this reporter vector into NHOKs without or with zoledronate (ZOL) or PAM.** $\mathrm{p}<0.01$.
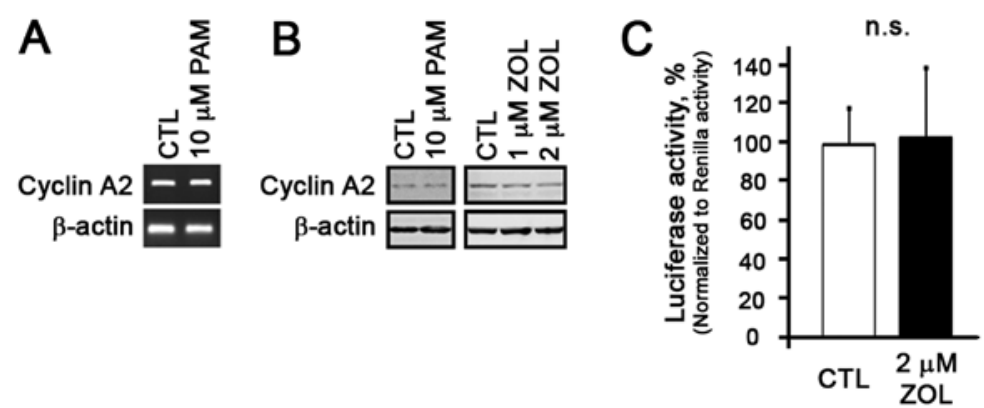

Figure 4. Bisphosphonates do not inhibit the expression of cyclin A2 in normal human oral fibroblasts (NHOFs). (A) NHOFs were treated with $10 \mu \mathrm{M}$ pamidronate (PAM) for 4 days, and the expression of cyclin A2 was examined by RT-qPCR. (B) NHOFs were treated with $10 \mu \mathrm{M}$ PAM for 4 days, or with 1 or $2 \mu \mathrm{M}$ zoledronate (ZOL) for 4 days. The expression of cyclin A2 was examined by western blot analysis. (C) Luciferase reporter assay was performed in NHOFs by transfecting this reporter vector in the presence of $2 \mu \mathrm{M}$ ZOL.n.s., not significant.

a cyclin A2-promoter luciferase reporter vector by cloning the 608 bp (-516 to +92) known to be essential for cyclin A2 promoter activity (Fig. 3B) $(12,13)$. The luciferase assay revealed a relatively 4-5-fold reduction in luciferase activity in the PAMand ZOL-treated NHOKs (Fig. 3C), indicating that cyclin A2 expression is indeed transcriptionally regulated by N-BPs.

$N$-BPs do not inhibit the expression of cyclin $A 2$ in normal human oral fibroblasts (NHOFs). Our previous study demonstrated that N-BPs at the lower concentration had no notable effects on the proliferation of NHOFs (9). Thus, in this study, in order to examine whether cyclin A2 expression is altered by N-BPs in NHOFs, we treated the NHOFs with N-BPs and observed no changes in cyclin $\mathrm{A} 2$ expression at both the mRNA and protein level (Fig. 4A and B). Cyclin A2 promoter luciferase reporter assay revealed that there was also no change in luciferase activity in the NHOFs treated with the N-BPs (Fig. 4C), indicating that $\mathrm{N}$-BPs at the concentrations which inhibited cyclin A2 in the NHOKs had no effects on cyclin A2 in the NHOFs.

\section{Discussion}

A significant body of evidence supports the notion that N-BPs cause epithelial toxicity. However, the underlying mechanisms are not yet fully understood. We previously demonstrated that bisphosphonates suppress proliferation without inducing the apoptosis of NHOKs (9). In this study, using microarray and IPA analyses, we found that N-BPs preferentially regulate genes associated with the cell cycle. Among the cell cycle-associated genes, the expression of cyclin A2 was specifically inhibited by the N-BPs in the NHOKs at the transcriptional level, and such an inhibition was not evident in the NHOFs, suggesting that the effects of BPs are cell type-specific. Furthermore, using oral epithelial tissue constructs, we demonstrated that BPs also cause the suppression of cyclin A2 in epithelial layers, indicating that epithelial toxicity by BPs is, at least in part, due to targeting cyclin A2 expression and suppressing cell proliferation.

$\mathrm{N}-\mathrm{BPs}$, derivatives of inorganic pyrophosphatase with a backbone of P-O-P, are stable and clinically applicable due to the replacement of oxygen molecule to the carbon molecule (P-C-P). This substitution enhances two independent, but synergistic biological properties of the bisphosphonates through the hydroxyl group (R1) and the nitrogen-containing group (R2) (14). The R1 group enhances the affinity to calciumenriched tissues, such as bone. On the other hand, the R2 group in BPs is the nitrogen-containing group, which is known to be responsible for an increase in biological potency by physically binding to and inhibiting the functions of farnesyl pyrophosphate synthase (FPPS) $(15,16)$. FPPS is the key branch-point enzyme in the mevalonate pathway required for generating a metabolite, farnesyl pyrophosphate (FPP), for the synthesis of cholesterol and protein prenylation. Prenylation involves the transfer of farnesyl and/or geranylgeranyl lipid groups onto the C-terminus of small GTPase proteins at the post-translational level $(17,18)$. As small GTPases, such as Ras, Rap1, Rab3A or RhoA are known to play pivotal roles in cellular functions, including proliferation, 
migration, morphology, polarity and secretion (19), BP-mediated changes in cellular functions may occur via the inhibition of the mevalonate pathway and protein prenylation.

A previous study found that the cyclin A2 gene was upregulated by $\mathrm{H}$-Ras and RhoA in their attempt to identify responsive genes by a group of different small GTPases (20). Of note, our recent study demonstrated that bisphosphonates inhibited cell cycle arrest at the $S$ phase (9), suggesting that some small GTPases such as H-Ras or RhoA may be targeted by bisphosphonates to regulate transcriptional machineries to suppress expression of the downstream genes, such as cyclin A2.

The precise role of transcriptional regulation by bisphosphonates on cyclin A2 has yet to be determined. Although small GTPases are known to mainly regulate cytoskeletal structures and functions, studies showed modulation of transcriptional expression by these small GTPases. For instance, Rac and Cdc42 induce the transcriptional activity of c-Jun by activating c-Jun aminoterminal kinawse (JNK), and Rho activates transcriptional activity of serum response factor (SRF) $(21,22)$. RalA has also been shown to activate urokinase plasminogen activator receptor (UPAR) via c-Src and AP1 (23). Thus, it is likely that bisphosphonates inhibit cyclin A2 expression by disrupting prenylation and transcriptionactivating functions of small GTPases.

It has been demonstrated that bisphosphonates have a potential role as putative cancer therapeutic agents. Bisphosphonate treatment induces the production of cytotoxic ATP analogs in tumor cells, leading to growth arrest (24). Bwisphosphonates also suppress angiogenesis via hypoxia-induction factor (HIF)-1 $\alpha /$ vascular endothelial growth factor (VEGF) signaling pathways in human breast cancer cells (25). Furthermore, bisphosphonates induce cell-cycle prolongation in murine lung cancer cells by alteration of cyclin and Ras expression (26). It has also been suggested that cyclin A2 may be the molecular target of the bisphosphonate-mediated suppression of cancer cells. Cyclin A2 is frequently found to be elevated in a variety of tumors (27). Ory et al showed that bisphosphonates inhibited the proliferation of osteosarcoma cells by inducing cell cycle arrest at the $\mathrm{S}$ and $\mathrm{G} 2 / \mathrm{M}$ phase $(28,29)$. In myeloma cells, bisphosphonates have also been shown to induce $S$ phase cell cycle arrest by activating the MAPK pathway (11). The direct association between bisphosphonates and cyclin A2 warrants further investigation.

In conclusion, in this study, we demonstrate that cyclin A2, which regulates the cell cycle at the $\mathrm{S}$ phase by forming a cyclin A2-Cdk2 complex, is inhibited by both bisphosphonates, PAM and ZOL, at the transcriptional level. BPs targeting cyclin A2 expression may serve as the dominant mode of the cell cycle arrest and the inhibition of cell proliferation. Our 3-dimensional (3D) oral mucosal tissue constructs also demonstrated that ZOL inhibited the expression of cyclin A2, suggesting that cyclin A 2 may be involved in bisphosphonate-induced epithelial toxicity by causing structural and architectural damages. The modulation of cyclin A2 may hold therapeutic potential to ameliorate epithelial toxicity mediated by bisphosphonates.

\section{Acknowledgements}

This study was supported by the grants from NIDCR/NIH R03DE021114, R01DE023348 and Dean's Faculty Research Seed grant to R.H.K.

\section{References}

1. Russell RG: Bisphosphonates: The first 40 years. Bone 49: 2-19, 2011.

2. Coxon FP, Thompson K and Rogers MJ: Recent advances in understanding the mechanism of action of bisphosphonates. Curr Opin Pharmacol 6: 307-312, 2006.

3. Reyes C, Hitz M, Prieto-Alhambra D and Abrahamsen B: Risks and benefits of bisphosphonate therapies. J Cell Biochem 117: 20-28, 2016.

4. Watts N, Freedholm D and Daifotis A: The clinical tolerability profile of alendronate. Int J Clin Pract Suppl 101: 51-61, 1999.

5. Rubegni $P$ and Fimiani $M$ : Images in clinical medicine. Bisphosphonate-associated contact stomatitis. N Engl J Med 355: e25, 2006

6. de Groen PC, Lubbe DF, Hirsch LJ, Daifotis A, Stephenson W, Freedholm D, Pryor-Tillotson S, Seleznick MJ, Pinkas H and Wang KK: Esophagitis associated with the use of alendronate. N Engl J Med 335: 1016-1021, 1996.

7. Proksch E, Brandner JM and Jensen JM: The skin: An indispensable barrier. Exp Dermatol 17: 1063-1072, 2008.

8. Bae S, Sun S, Aghaloo T, Oh JE, McKenna CE, Kang MK, Shin KH, Tetradis S, Park NH and Kim RH: Development of oral osteomucosal tissue constructs in vitro and localization of fluorescently-labeled bisphosphonates to hard and soft tissue. Int J Mol Med 34: 559-563, 2014.

9. Kim RH, Lee RS, Williams D, Bae S, Woo J, Lieberman M, Oh JE, Dong Q, Shin KH, Kang MK, et al: Bisphosphonates induce senescence in normal human oral keratinocytes. J Dent Res 90: 810-816, 2011.

10. Green JR: Chemical and biological prerequisites for novel bisphosphonate molecules: Results of comparative preclinical studies. Semin Oncol 28 (Suppl 6): 4-10, 2001.

11. Iguchi T, Miyakawa Y, Yamamoto K, Kizaki M and Ikeda Y: Nitrogen-containing bisphosphonates induce S-phase cell cycle arrest and apoptosis of myeloma cells by activating MAPK pathway and inhibiting mevalonate pathway. Cell Signal 15: 719-727, 2003.

12. Horiuchi K, Umetani M, Minami T, Okayama H, Takada S, Yamamoto M, Aburatani H, Reid PC, Housman DE, Hamakubo T, et al: Wilms' tumor 1-associating protein regulates G2/M transition through stabilization of cyclin A2 mRNA. Proc Natl Acad Sci USA 103: 17278-17283, 2006.

13. Henglein B, Chenivesse X, Wang J, Eick D and Bréchot C: Structure and cell cycle-regulated transcription of the human cyclin A gene. Proc Natl Acad Sci USA 91: 5490-5494, 1994.

14. Reszka AA and Rodan GA: Nitrogen-containing bisphosphonate mechanism of action. Mini Rev Med Chem 4: 711-719, 2004.

15. Bergstrom JD, Bostedor RG, Masarachia PJ, Reszka AA and Rodan G: Alendronate is a specific, nanomolar inhibitor of farnesyl diphosphate synthase. Arch Biochem Biophys 373: 231-241, 2000.

16. van Beek E, Pieterman E, Cohen L, Löwik C and Papapoulos S: Nitrogen-containing bisphosphonates inhibit isopentenyl pyrophosphate isomerase/farnesyl pyrophosphate synthase activity with relative potencies corresponding to their antiresorptive potencies in vitro and in vivo. Biochem Biophys Res Commun 255: 491-494, 1999.

17. Sinensky M: Recent advances in the study of prenylated proteins. Biochim Biophys Acta 1484: 93-106, 2000.

18. Williams CL: The polybasic region of Ras and Rho family small GTPases: A regulator of protein interactions and membrane association and a site of nuclear localization signal sequences. Cell Signal 15: 1071-1080, 2003.

19. Csépányi-Kömi R, Lévay M and Ligeti E: Small G proteins and their regulators in cellular signalling. Mol Cell Endocrinol 353: $10-20,2012$.

20. Teramoto H, Malek RL, Behbahani B, Castellone MD, Lee NH and Gutkind JS: Identification of H-Ras, RhoA, Racl and Cdc42 responsive genes. Oncogene 22: 2689-2697, 2003.

21. Coso OA, Chiariello M, Yu JC, Teramoto H, Crespo P, Xu N, Miki T and Gutkind JS: The small GTP-binding proteins Rac1 and $\mathrm{Cdc} 42$ regulate the activity of the JNK/SAPK signaling pathway. Cell 81: 1137-1146, 1995.

22. Hill CS, Wynne J and Treisman R: The Rho family GTPases RhoA, Racl, and CDC42Hs regulate transcriptional activation by SRF. Cell 81: 1159-1170, 1995.

23. Okan E, Drewett V, Shaw PE and Jones P: The small-GTPase RalA activates transcription of the urokinase plasminogen activator receptor (uPAR) gene via an AP1-dependent mechanism. Oncogene 20: 1816-1824, 2001. 
24. Mönkkönen H, Kuokkanen J, Holen I, Evans A, Lefley DV, Jauhiainen M, Auriola S and Mönkkönen J: Bisphosphonateinduced ATP analog formation and its effect on inhibition of cancer cell growth. Anticancer Drugs 19: 391-399, 2008.

25. Sonnemann J, Eckervogt V, Truckenbrod B, Boos J, Winkelmann W and van Valen F: The bisphosphonate pamidronate is a potent inhibitor of Ewing's sarcoma cell growth in vitro. Anticancer Drugs 14: 767-771, 2003.

26. Li YY, Chang JW, Liu YC, Wang CH, Chang HJ, Tsai MC, Su SP and Yeh KY: Zoledronic acid induces cell-cycle prolongation in murine lung cancer cells by perturbing cyclin and Ras expression. Anticancer Drugs 22: 89-98, 2011.

27. Yam CH, Fung TK and Poon RY: Cyclin A in cell cycle control and cancer. Cell Mol Life Sci 59: 1317-1326, 2002.
28. Iguchi T, Miyakawa Y, Saito K, Nakabayashi C, Nakanishi M, Saya H, Ikeda Y and Kizaki M: Zoledronate-induced S phase arrest and apoptosis accompanied by DNA damage and activation of the ATM/Chk1/cdc25 pathway in human osteosarcoma cells. Int J Oncol 31: 285-291, 2007.

29. Ory B, Blanchard F, Battaglia S, Gouin F, Rédini F and Heymann D: Zoledronic acid activates the DNA S-phase checkpoint and induces osteosarcoma cell death characterized by apoptosis-inducing factor and endonuclease- $\mathrm{G}$ translocation independently of p53 and retinoblastoma status. Mol Pharmacol 71: 333-343, 2007. 